\title{
Associations of Telehealth Care Delivery with Pediatric Health Care Provider Well-Being
}

\author{
Richelle deMayo ${ }^{1,2,3}$ Yungui Huang ${ }^{4}$ En-Ju D. Lin ${ }^{4}$ Jennifer A. Lee ${ }^{5,6,7}$ Andrew Heggland ${ }^{1,3,8}$
} Jane $\mathrm{Im}^{1,3,9}$ Christopher Grindle ${ }^{1,10,11}$ Aarti Chandawarkar ${ }^{5,7}$

${ }^{1}$ Division of Clinical Informatics, Connecticut Children's Medical Center, Hartford, Connecticut, United States

2 Division of Pain and Palliative Medicine, Connecticut Children's Medical Center, Hartford, Connecticut, United States

${ }^{3}$ Department of Pediatrics, University of Connecticut School of Medicine, Farmington, Connecticut, United States

${ }^{4}$ Research Information Solutions and Innovation, The Abigail Wexner Research Institute, Nationwide Children's Hospital, Columbus, Ohio, United States

${ }^{5}$ Division of Clinical Informatics, Nationwide Children's Hospital, Columbus, Ohio, United States

${ }^{6}$ Division of Pediatric Gastroenterology, Hepatology and Nutrition, Nationwide Children's Hospital, Columbus, Ohio, United States

7 Department of Pediatrics, The Ohio State University College of Medicine, Columbus, Ohio, United States

8 Division of Pediatric Emergency Medicine, Connecticut Children's Medical Center, Hartford, Connecticut, United States

${ }^{9}$ Division of Pediatric Hospital Medicine, Connecticut Children's Medical Center, Hartford, Connecticut, United States
Address for correspondence Richelle deMayo, MD, CM, Department of Pediatrics, University of Connecticut, 282 Washington Avenue, Hartford, CT 06106, United States

(e-mail: rdemayo@connecticutchildrens.org).

\footnotetext{
${ }^{10}$ Department of Surgery, University of Connecticut School of Medicine, Farmington, Connecticut, United States

${ }^{11}$ Division of Otolaryngology, Connecticut Children's Medical Center, Hartford, Connecticut, United States
} Appl Clin Inform 2022;13:230-241.

\section{Abstract}

Keywords

- telemedicine and telehealth

- burnout, professional

- efficiency

- clinical effectiveness

- personal satisfaction
Background The rapid, large-scale deployment of new health technologies can introduce challenges to clinicians who are already under stress. The novel coronavirus disease 19 (COVID-19) pandemic transformed health care in the United States to include a telehealth model of care delivery. Clarifying paths through which telehealth technology use is associated with change in provider well-being and interest in sustaining virtual care delivery can inform planning and optimization efforts.

Objective This study aimed to characterize provider-reported changes in well-being and daily work associated with the pandemic-accelerated expansion of telehealth and assess the relationship of provider perceptions of telehealth effectiveness, efficiency, and work-life balance with desire for future telehealth.

Methods A cross-sectional survey study was conducted October through November 2020, 6 months after the outbreak of COVID-19 at three children's hospitals. Factor analysis and structural equation modeling (SEM) were used to examine telehealth factors associated with reported change in well-being and desire for future telehealth. Results A total of 947 nontrainee physicians, advanced practice providers, and psychologists were surveyed. Of them, 502 (53.0\%) providers responded and 467 received

July 10,2021

accepted after revision

December 14, 2021
DOI https://doi.org/

$10.1055 / \mathrm{s}-0042-1742627$.

ISSN 1869-0327.

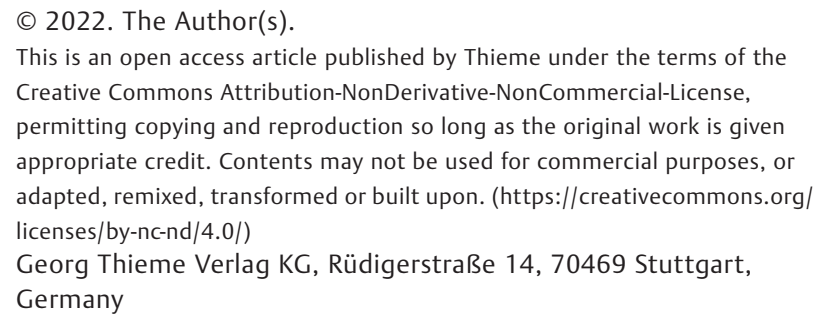


(49.3\%) met inclusion criteria of telehealth use during the study period. Of these, 325 (69.6\%) were female, 301 (65.6\%) were physicians, and 220 (47.1\%) were medical subspecialists. Providers were 4.77 times as likely (95\% confidence interval [CI]: 3.297.06) to report improved versus worsened well-being associated with telehealth. Also, 95.5\% of providers (95\% Cl: $93.2-97.2 \%$ ) wish to continue performing telehealth postpandemic. Our model explains $66 \%$ of the variance in telehealth-attributed provider well-being and $59 \%$ of the variance for future telehealth preference and suggests telehealth resources significantly influence provider-perceived telehealth care effectiveness which in turn significantly influences provider well-being and desire to perform telehealth.

Conclusion Telehealth has potential to promote provider well-being; telehealthrelated changes in provider well-being are associated with both provider-perceived effectiveness of telemedicine for patients and adequacy of telehealth resources.

\section{Background and Significance}

The novel coronavirus disease 19 (COVID-19) pandemic created opportunities and imperatives to introduce and expand telehealth as quickly as possible. Health services across the United States were swiftly transformed to virtual care delivery, often within a few weeks, encouraged by federal and state emergency actions. ${ }^{1}$ Prepandemic, $15 \%$ of physicians offered telehealth services and $10 \%$ of patients had participated in a telehealth encounter. Between spring and summer $2020,85 \%$ of physicians and $46 \%$ of patients reported use of telehealth. ${ }^{2}$

Physician stress and burnout, particularly associated with technology use, were issues of considerable interest prepandemic. $^{3-7}$ In the context of a public health crisis that placed extraordinary responsibilities on health care professionals and contributed to deterioration of work-life happiness, clinician well-being has become an even greater concern. ${ }^{8,9}$ As telehealth adoption and availability grows, so does the need to understand providers' perceptions of its benefits, burdens, and barriers.

Few studies have examined the impact of telehealth on provider well-being. Limited evidence from the pre-COVID19 era on telehealth user and provider wellness and attitudes, largely focused on hospital-based providers, found telehealth decreased on-call travel burdens of physicians and improved their perceptions of teamwork and safety climate. ${ }^{10,11}$ Other studies highlighted the salience of usability and effectiveness for physicians' willingness to adopt telehealth and suggested variability in attitudes between telehealth-experienced and telehealth-inexperienced providers. ${ }^{12,13}$

This study was guided by a National Academies of Medicine (NAM) conceptual model that links cross-disciplinary clinician resilience and well-being with outcomes for health care professionals, patients, and the broader health system. ${ }^{14}$ Like other frameworks of workplace well-being, this model incorporates individual and environmental inputs. It proposes clinician well-being is determined by a balance between demands and resources, with external system factors more influential than internal individual ones. The model consciously situates the health care professional, patient relationship at its center.

The terms "telemedicine" and "telehealth" have been defined in many ways. ${ }^{15}$ For this manuscript, we employ the term "telehealth" to refer to synchronous encounters between providers and patients, inclusive of video plus audio, as well as audio only (telephonic) interactions.

\section{Objective}

This study sought to describe provider-reported changes in daily work and well-being, accompanying the rapid expansion of telehealth for health care delivery. Provider perceptions of telehealth effectiveness, efficiency, and work-life balance were examined to identify factors associated with improved provider well-being and provider desire for future telehealth.

\section{Methods}

\section{Setting}

A cross-sectional observational survey study was conducted in fall 2020 by three pediatric health care systems in the United States: Connecticut Children's (CC) Hospital, Nationwide Children's Hospital (NCH), and East Tennessee Children's Hospital (ETCH). These institutions represent two academic tertiary care medical centers (CC: 187 beds and 400,000 annual outpatient visits; and NCH: 625 beds and 1.3 million annual outpatient visits), and one community hospital (ETCH: 152 beds and 120,000 annual outpatient visits). The two larger institutions use an enterprise Epic Electronic Health Record (EHR) platform (Epic Systems Corporation, Verona, Wisconsin, United States) with integrated Zoom video functionality (Zoom Video Communications, San Jose, California, United States); the smallest institution uses a Meditech EHR system (Medical Information Technology Inc., Westwood, Massachusetts, United States) with a nonintegrated Zoom video platform. The study protocol was 
approved by the Institutional Review Boards (IRB) at CC and $\mathrm{NCH}$ and was deemed exempt by the IRB at ETCH.

\section{Survey Design and Sampling}

Since most existing telemedicine instruments are intended to assess the patient experience, a brief platform-agnostic provider experience questionnaire was developed by the collaborative research team to inform organizational strategic plans for continuous improvement in telehealth. ${ }^{16}$ The questionnaire, which is freely available, ${ }^{17}$ drew upon findings of a 2016 American Academy of Pediatrics survey of pediatrician telehealth attitudes and experience. ${ }^{18,19}$ Items were adapted from existing validated clinician technology user and patient telemedicine experience instruments, including the KLAS Electronic Medical Records User Experience Survey, the Telemedicine Satisfaction Questionnaire, and the Telehealth Usability Questionnaire. ${ }^{20-22}$ Novel items were created in domains of telehealth demands, resources, benefits, and barriers. The questionnaire collected demographic information and a self-reported burnout measure previously shown to correlate with the Maslach Burnout Inventory's emotional exhaustion subscale. ${ }^{23}$ The survey was pilot tested with 10 providers who suggested edits and confirmed time burden and comprehensibility of the final version.

The survey was deployed using REDCap version 10.6.10 software (Vanderbilt University, Nashville, Tennessee, United States) via e-mail to a list-based population of 947 primary care and subspecialty health providers, excluding trainees, at the three children's hospitals. NCH chose to include psychology providers, while CC and East Tennessee did not. Consent to participate was implied by survey completion. The provider population for this analysis was limited to physicians, advanced practice nurses, physician assistants, and psychologists who reported active engagement in telehealth between March 1, 2020, and October 31, 2020. Statistical analysis was completed in SPSS version 27 (IBM Corporation) and in R 4.0.2 (R Core Team), using the polychor, lavaan, and psych packages. ${ }^{24-26}$

Provider telehealth-associated well-being was operationalized as the 6-point Likert scale response to "How has the introduction/expansion of telemedicine affected your professional well-being?" with responses of "improved" or "greatly improved" considered positive attributed change. Preference for future telehealth was captured as a 5-point Likert's scale response to "In an ideal world, how much telemedicine would you provide after COVID-19?" Burnout was determined via a five-choice single-item validated measure that has been extensively described in previous literature. 27,28

\section{Statistical Analysis}

Survey responses were analyzed as standard summary statistics, overall, and by respondent organization to assess for institutional effect. Logistic regression was used to estimate the odds of provider-reported improved telehealth-attributed well-being and the odds of provider-reported desire for future telehealth with demographic factors and responses regarding telehealth resources, demands, benefits, and barriers. Analyses made maximal use of available data by omitting missing values by pairwise deletion. Factor analysis and structural equation modeling were performed to incorporate multiple independent variables, extract latent constructs, and simultaneously analyze interrelationships with multiple outcomes of interest. Well-being, future telehealth preference, and burnout were treated as binary and ordered categorical variables without substantive difference between methods. Narrative comments were examined inductively and coded to generate leading qualitative themes by members of the study team.

\section{Results}

Survey response rate was 53.0\%. Of 502 respondents, 467 (93.0\%) met inclusion criteria by reporting active engagement in telehealth between March and October 2020. Responding providers were not statistically different from nonresponding providers in terms of gender or role. Also, 458 respondents (98.1\%) answered at least 9 of 10 designated "core" survey questions and 105 (22.5\%) supplied additional narrative comments. Core survey questions included responses to items about telehealth use, change in wellbeing attributed to telehealth, and information about role, gender, specialty, career stage, and clinical practice setting. Providers volunteered 69 free-text comments regarding telehealth benefits and 57 comments on telehealth barriers and burdens.

Respondent demographics are summarized in -Table 1.

\section{Telehealth Usage}

Most survey respondents were telehealth-naïve, having no previous telehealth experience, prior to COVID-19. For all institutions, median provider telehealth experience at baseline was $0 \%$ (95\% confidence interval $[\mathrm{CI}]$ : 0.0-0.0\%). Providers experienced dramatic changes in daily work as they rapidly adopted telehealth; median peak telehealth use during the time period between the outbreak of COVID-19 and the survey across all institutions was 77\% (95\% CI: 75.0$80.0 \%$ ) of patient interactions. At ETCH, providers did not rely as heavily on telehealth as the larger institutions; median peak usage there was 7.0\% (95\% CI: 5.0-22.5\%). The telehealth transformation at participating institutions was largely focused on ambulatory settings. $\mathrm{NCH}$ was not using telehealth in their emergency or inpatient areas at the time of survey. Median peak telehealth use for exclusively hospital-based acute care physicians was 50.0\% (95\% CI: $2.0-79.0 \%$ ) of patient encounters.

\section{Provider Well-Being and Desire for Future Telehealth} A total of 378 of 388 (83.1\%) providers characterized the shift to telehealth as impactful to well-being. Over one-third of respondents, 35.8\% (167/388), described "improved or greatly improved" telehealth-associated well-being. Respondents were 4.77 times as likely (95\% CI: 3.44-7.26) to characterize telehealth-related changes in well-being as positive versus negative. Gender, role, career stage, practice setting, and 
Table 1 Survey respondent demographics

\begin{tabular}{|c|c|c|c|c|c|c|}
\hline & & $\begin{array}{l}\text { CC } \\
\text { (no.) }\end{array}$ & $\begin{array}{l}\text { ETCH } \\
\text { (no.) }\end{array}$ & $\begin{array}{l}\mathrm{NCH} \\
\text { (no.) }\end{array}$ & $\begin{array}{l}\text { Overall } \\
\text { (no.) }\end{array}$ & $\begin{array}{l}\text { Percent } \\
\text { of total (\%) }\end{array}$ \\
\hline \multirow[t]{3}{*}{ Gender } & Female & 77 & 28 & 220 & 325 & 70 \\
\hline & Male & 40 & 10 & 87 & 137 & 29 \\
\hline & Other/not disclosed & 2 & 0 & 3 & 5 & 1 \\
\hline \multirow[t]{3}{*}{ Role } & Attending physician & 87 & 26 & 188 & 301 & 66 \\
\hline & Advanced practice provider & 26 & 10 & 75 & 111 & 24 \\
\hline & Psychologist & 0 & 0 & 47 & 47 & 10 \\
\hline \multirow[t]{3}{*}{ Career stage } & $0-10$ years & 43 & 10 & 131 & 184 & 39 \\
\hline & $11-20$ years & 38 & 9 & 100 & 147 & 32 \\
\hline & $21+$ years & 38 & 19 & 79 & 136 & 29 \\
\hline \multirow[t]{6}{*}{ Specialty } & Medical subspecialist & 52 & 11 & 157 & 220 & 47 \\
\hline & Surgical subspecialist & 26 & 1 & 41 & 68 & 15 \\
\hline & Primary care & 7 & 15 & 32 & 54 & 12 \\
\hline & Hospital care/critical care & 12 & 1 & 11 & 24 & 5 \\
\hline & Psychiatry/psychology & 2 & 0 & 62 & 64 & 14 \\
\hline & Other/unable to be determined & 18 & 8 & 2 & 28 & 6 \\
\hline \multirow[t]{4}{*}{ Practice setting } & Outpatient & 34 & 23 & 141 & 198 & 42 \\
\hline & Inpatient & 3 & 0 & 1 & 4 & 1 \\
\hline & ED/urgent care & 0 & 0 & 1 & 1 & $<0.1$ \\
\hline & Multiple settings & 82 & 15 & 167 & 264 & 57 \\
\hline \multirow[t]{5}{*}{ Burnout } & $\begin{array}{l}\text { No symptoms of stress } \\
\text { or burnout }\end{array}$ & 25 & 10 & 56 & 91 & 20 \\
\hline & Stress but no burnout & 56 & 16 & 149 & 221 & 48 \\
\hline & Burnout symptoms & 28 & 9 & 80 & 117 & 25 \\
\hline & Persistent burnout symptoms & 6 & 2 & 21 & 29 & 6 \\
\hline & Complete burnout & 0 & 0 & 4 & 4 & 1 \\
\hline $\begin{array}{l}\text { Pre-COVID-19 } \\
\text { telehealth } \\
\text { experience }\end{array}$ & Telehealth Naive & 108 & 31 & 223 & 362 & 72 \\
\hline
\end{tabular}

Abbreviations: CC, Connecticut Children's Hospital; COVID-19, novel coronavirus disease 2019; ECTH, East Tennessee Children's Hospital; ED, emergency department; NCH, Nationwide Children's Hospital.

institution were not significantly related to positive wellbeing ratings. Surgical providers were significantly less likely than behavioral health specialty providers to report improved well-being (odds ratio $[\mathrm{OR}]=0.46$; 95\% CI: $0.22-$ 0.96). Providers with any degree of burnout were significantly less likely to report improved telehealth-attributed well-being ( $\mathrm{OR}=0.53$; 95\% CI: 0.34-0.081). Absent burnout symptoms, there was no statistically significant difference in likelihood of improved telehealth-associated well-being between providers who denied and those who endorsed professional stress.

Also, 411 of 467 (95.5\%) providers wished to continue telehealth post-COVID-19, with most, (317/467 [67.9\%]), preferring to perform at least "some" telehealth. Provider role and specialty were significantly associated with desire for postpandemic telehealth (-Table 2). Providers with improved telehealth-attributed well-being were 9.96 times as likely (95\% CI: $5.41-18.32$ ) to prefer some or more telehealth going forward.

\section{Telehealth Successes from a Provider Perspective}

There was widespread provider consensus of the usefulness of telehealth for clinical care delivery. Furthermore, 60.7\% (283/467) described themselves as "mostly" or "completely" able to deliver high quality care through telehealth. Only 4 of 467 respondents $(0.9 \%)$ reported telehealth is "not at all able" to meet their patient needs. Also, 67.5\% (315/467) reported they can "mostly" or "completely" meet patient needs through telehealth. Again, 73.0\% (341/467) also felt "mostly" or "completely" able to engage meaningfully with patients via telehealth. Moreover, 84.8\% (396/467) do not believe virtual care undermines the provider-patient relationship, reporting telehealth had a positive or net neutral influence on the provider-patient relationship. 
Table 2 Univariable binary logistic regression models of improved well-being and preference for future telehealth

\begin{tabular}{|c|c|c|c|c|c|c|c|c|c|c|}
\hline \multirow{3}{*}{$\begin{array}{l}\text { Characteristic } \\
\text { Gender }\end{array}$} & \multicolumn{5}{|c|}{ Telehealth-attributed improved well-being } & \multicolumn{5}{|c|}{$\begin{array}{l}\text { Preference for some or greater future } \\
\text { telehealth }\end{array}$} \\
\hline & \multirow[t]{2}{*}{ OR } & \multicolumn{2}{|c|}{$95 \% \mathrm{Cl}$} & \multirow[t]{2}{*}{$p$-Value } & \multirow[t]{2}{*}{$R^{2}(\%)$} & \multirow[t]{2}{*}{ OR } & \multicolumn{2}{|c|}{$95 \% \mathrm{Cl}$} & \multirow[t]{2}{*}{$p$-Value } & \multirow[t]{2}{*}{$R^{2}(\%)$} \\
\hline & & & & & & & & & & \\
\hline Female & 0.86 & 0.14 & 5.19 & 0.865 & \multirow[t]{3}{*}{0} & 1.57 & 0.26 & 9.53 & 0.626 & \multirow[t]{3}{*}{1} \\
\hline Male & 0.78 & 0.13 & 4.85 & 0.793 & & 1.12 & 0.18 & 6.96 & 0.900 & \\
\hline Other/nondisclosed & 1 (reference) & & & & & 1 (reference) & & & & \\
\hline \multicolumn{11}{|l|}{ Role } \\
\hline Physician & 0.81 & 0.43 & 1.54 & 0.524 & \multirow[t]{4}{*}{2} & 0.13 & 0.04 & 0.43 & $<0.001$ & \multirow[t]{4}{*}{4} \\
\hline APRN & 1.12 & 0.55 & 2.28 & 0.755 & & 0.12 & 0.04 & 0.42 & $<0.001$ & \\
\hline PA & 0.16 & 0.02 & 1.37 & 0.094 & & 0.08 & 0.02 & 0.43 & 0.003 & \\
\hline Psychologist & 1 (reference) & & & & & 1 (reference) & & & & \\
\hline \multicolumn{11}{|l|}{ Specialty } \\
\hline Medical subspecialist & 0.88 & 0.50 & 1.55 & 0.656 & \multirow[t]{5}{*}{3} & 0.16 & 0.06 & 0.46 & $<0.001$ & \multirow[t]{5}{*}{7} \\
\hline Surgical subspecialist & 0.46 & 0.22 & 0.96 & 0.038 & & 0.07 & 0.02 & 0.22 & $<0.001$ & \\
\hline Primary care & 0.48 & 0.22 & 1.05 & 0.067 & & 0.10 & 0.03 & 0.31 & $<0.001$ & \\
\hline Hospitalist & 0.69 & 0.26 & 1.83 & 0.451 & & 0.07 & 0.02 & 0.24 & $<0.001$ & \\
\hline $\begin{array}{l}\text { Behavioral health } \\
\text { specialist }\end{array}$ & 1 (reference) & & & & & 1 (reference) & & & & \\
\hline \multicolumn{11}{|l|}{ Career stage } \\
\hline Late $(21+$ years $)$ & 0.97 & 0.61 & 1.56 & 0.909 & \multirow[t]{3}{*}{1} & 0.83 & 0.52 & 1.33 & 0.435 & \multirow[t]{3}{*}{0} \\
\hline Mid (11-20 years) & 1.36 & 0.87 & 2.13 & 0.183 & & 0.93 & 0.58 & 1.49 & 0.764 & \\
\hline Early ( $<10$ years) & 1 (reference) & & & & & 1 (reference) & & & & \\
\hline \multicolumn{11}{|l|}{ Practice setting } \\
\hline Ambulatory & 0.95 & 0.65 & 1.39 & 0.780 & \multirow[t]{2}{*}{0} & 1.16 & 0.78 & 1.73 & 0.470 & \multirow[t]{2}{*}{0} \\
\hline Combined & 1 (reference) & & & & & 1 (reference) & & & & \\
\hline \multicolumn{11}{|l|}{ Stress and burnout } \\
\hline No stress or burnout & 2.33 & 1.35 & 4.05 & 0.003 & \multirow[t]{3}{*}{3} & 0.77 & 0.44 & 1.32 & 0.337 & \multirow[t]{3}{*}{0} \\
\hline Stress but no burnout & 1.74 & 1.11 & 2.75 & 0.016 & & 1.01 & 0.64 & 1.57 & 0.982 & \\
\hline$>1$ burnout symptoms & 1 (reference) & & & & & 1 (reference) & & & & \\
\hline
\end{tabular}

Abbreviations: APRN, advanced practice nurse; $\mathrm{Cl}$, confidence interval; OR, odds ratio; PA, physician assistant.

Prompted to describe the benefits of telehealth versus inperson care "to you as a provider," respondents most commonly endorsed ways in which telehealth enables them to expand access to patients (341/467 [73.0\%]) and renews their focus on patient-centric care (228/467 [48.8\%]). Providers also indicated their own work-life balance (flexibility or control) was a leading benefit 204/467 [43.7\%]).

Providers' free text comments described benefits that mapped to themes of improved patient-centered care, improved quality and safety, decreased no-show rates, and personal convenience.

\section{Provider-Identified Telehealth Stressors and Shortcomings}

Across the three organizations, provider respondents identified emergent telehealth demands not matched by available telehealth resources as presented in - Fig. 1. Despite differ- ences in platforms and implementation at participating hospitals, $85 \%$ of providers (397/467) named patient and/or provider technology problems (devices, connectivity, sound/image quality, and patient portal issues) as a barrier to effective use of telehealth. Of these, 72.6\% (339/467) identified novel technology burdens for providers or patients and $52.7 \%$ (245/465) reported inadequate technical support for themselves and their patients.

At all organizations, training was a universal unmet need; regardless of institutional curriculum or teaching modality, $56.8 \%$ of providers $(264 / 465)$ rated telehealth training less than "good."

Further, $43.1 \%$ of providers (200/464) noted inadequate clinical support for telehealth encounters, and $32.5 \%$ (152/467) described inefficiencies in telehealth clinical processes. Only 9.6\% (45/467) of respondents agreed "collaboration" was a benefit of telehealth, suggesting workflows 


\section{Telehealth resource adequacy}

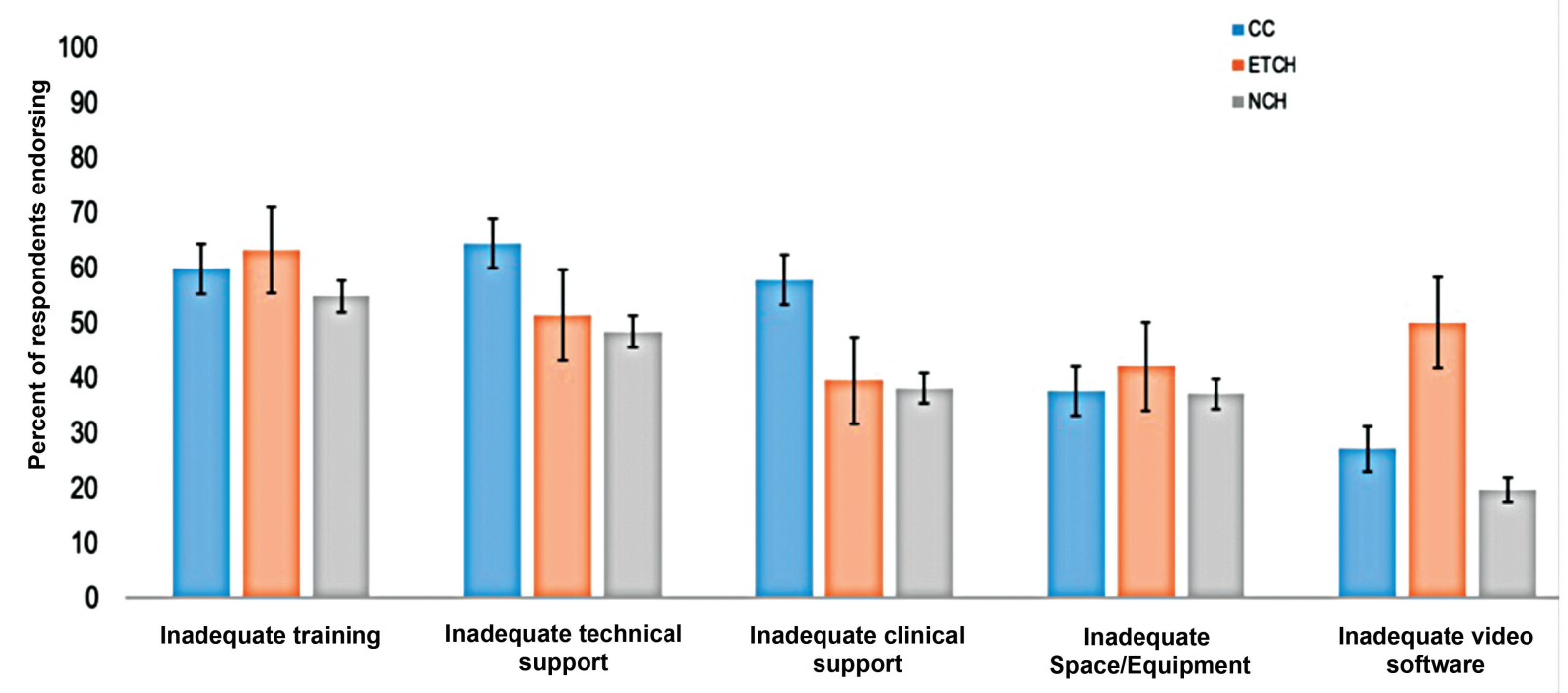

Fig. 1 Provider-reported telehealth resource adequacy. CC, Connecticut Children's Hospital; ECTH, East Tennessee Children's Hospital; NCH, Nationwide Children's Hospital.

that foster effective teamwork are lacking. More than half of providers (298/467 [63.8\%]), identified challenges in obtaining an adequate physical exam via telehealth. Providers also described difficulties determining which patients would be well-served by telehealth.

Aldo, $37.6 \%$ of respondents (175/465) reported infrastructure inadequacies including provider space and equipment for telehealth. Video software shortcomings were identified as a top unmet need at one organization.

Providers also offered additional details in their narrative comments regarding leading telehealth stressors which indicted workflow inefficiencies, technical problems, and challenges regarding the appropriateness of telehealth for particular patients. Provider-reported telehealth benefits and barriers/burdens are presented in -Fig. 2 .

\section{Factors Associated with Improved Well-Being and Desire to Continue Telehealth}

The results of univariate logistic regression modeling of positive telehealth-attributed well-being and preference for substantial future health with provider-reported benefits, barriers, and burdens are presented in -Table 3 .

To better understand the interplays among telehealth characteristics and outcomes of interest, we performed factor analysis and structural equation modeling to reduce data dimensionality while preserving variable information and accounting for correlations. Six latent factors were initially identified through exploratory factor analysis and three were retained after confirmatory analysis. "Telehealth Patient Effectiveness" includes three variables: (1) the abilities to engage with patients meaningfully, (2) serve patients' needs, and (3) deliver high quality care. "Telehealth Provider Satisfaction" includes three variables that capture providers' opinions of telehealth's impact on their own well-being and the provider-patient relationship, as well as the desire for continued telehealth. "Telehealth Resources" includes five variables related to the adequacy of infrastructure as follows: (1) training, (2) space/equipment, (3) video software, (4) clinical support, and (5) technical support. These three factors account for $66 \%$ of the variance in provider wellbeing and $59 \%$ of the variance in provider desire for future telehealth.

Each factor has high internal consistency with composite reliability of $0.91,0.89$, and 0.82 , exceeding the recommended level of $0.70,{ }^{29}$ and average variance extracted (AVE) of $0.78,0.62$, and 0.61 , exceeding the recommended level of $0.50,{ }^{30}$ respectively, for patient effectiveness, resources, and provider satisfaction. The absence of variable crossloadings and moderate between factor correlations of 0.66 ("Provider Satisfaction" and "Patient Effectiveness"), 0.41 ("Patient Effectiveness" and "Resources"), and 0.37 ("Provider Satisfaction" and "Resources") suggest each factor is distinct enough to be a separate construct.

Structural equation modeling revealed significant relationships between "Resources" and "Patient Effectiveness" and between "Provider Satisfaction" and "Patient Effectiveness." "Resources" are related to "Provider Satisfaction" in a statistically significant way, but the direct relationship is much weaker than the indirect one through "Patient Effectiveness" (i.e., "Resources" to "Patient Effectiveness" then to "Provider Satisfaction"). - Figs. 3 and 4 show the structural model results with estimated path coefficients and residual variance.

Path analysis revealed an indirect relationship between "Resources" and "Provider Desire for Future Telehealth." There was a statistically significant direct relationship between "Resources" and "Provider Burnout." There was not a 


\section{Provider-perceived telehealth benefits}

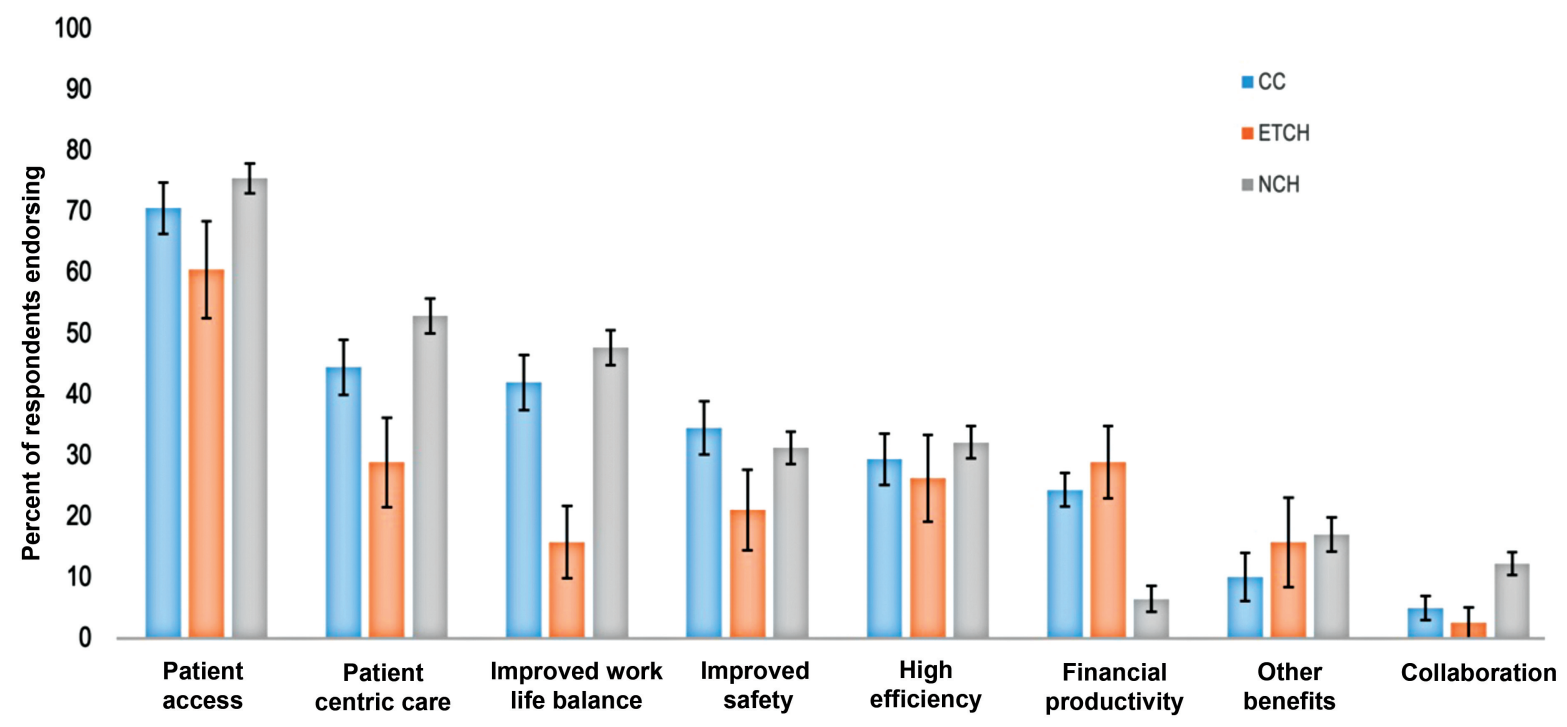

\section{Provider-perceived telehealth barriers}

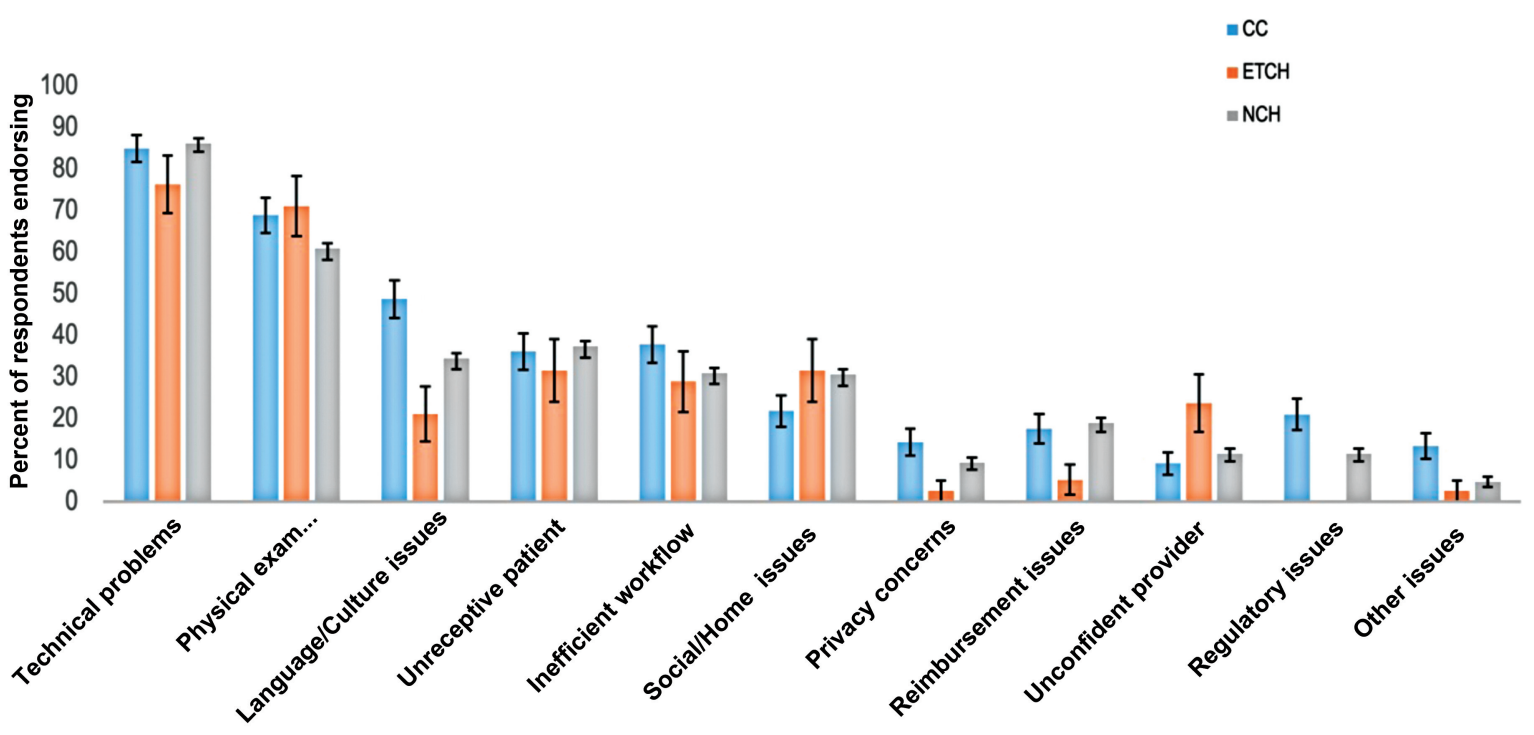

Fig. 2 Provider-reported telehealth barriers and benefits. CC, Connecticut Children's Hospital; ECTH, East Tennessee Children's Hospital; NCH, Nationwide Children's Hospital.

statistically significant relationship between perceived "Patient Effectiveness" and "Provider Burnout."

\section{Discussion}

The Pediatric Provider Telehealth Work and Well-being Survey offers one of the first large scale assessments to focus on the provider telehealth experience and associations between telehealth use and provider well-being. Our findings identify potential benefits of telehealth for provider well- being across roles and specialties. Structural equation modeling demonstrates provider perception of patient telehealth effectiveness that is significantly associated with a provider's telehealth-attributable well-being. Consistent with "Patient Well-Being" and the "Clinician-Patient Relationship" as central constructs in the NAM model, our findings indicate telehealth's capability to engage patients to meet their needs and enable delivery of high-quality care contributes importantly to provider satisfaction with telehealth. Path analysis reveals close ties between the patient- 


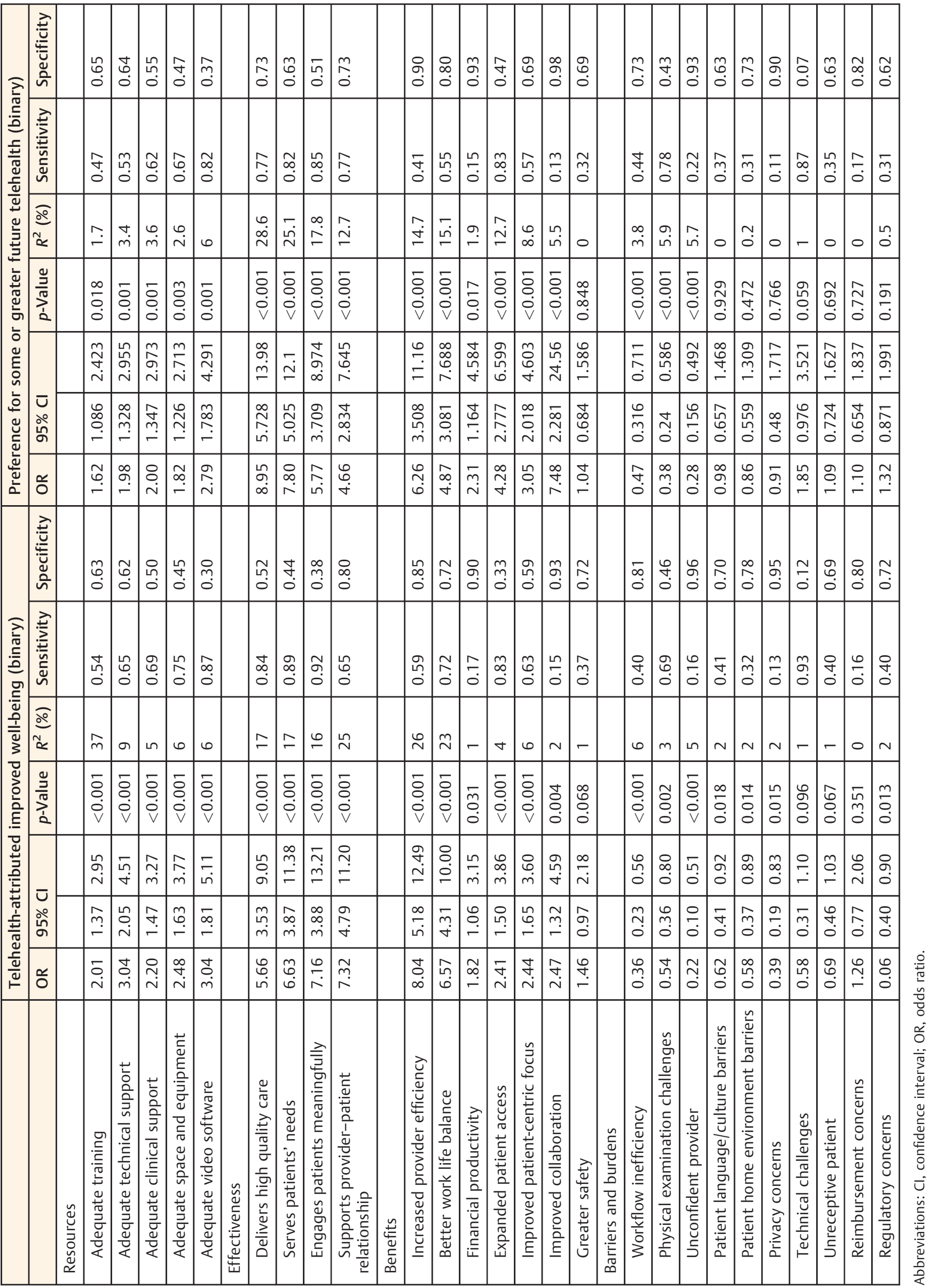




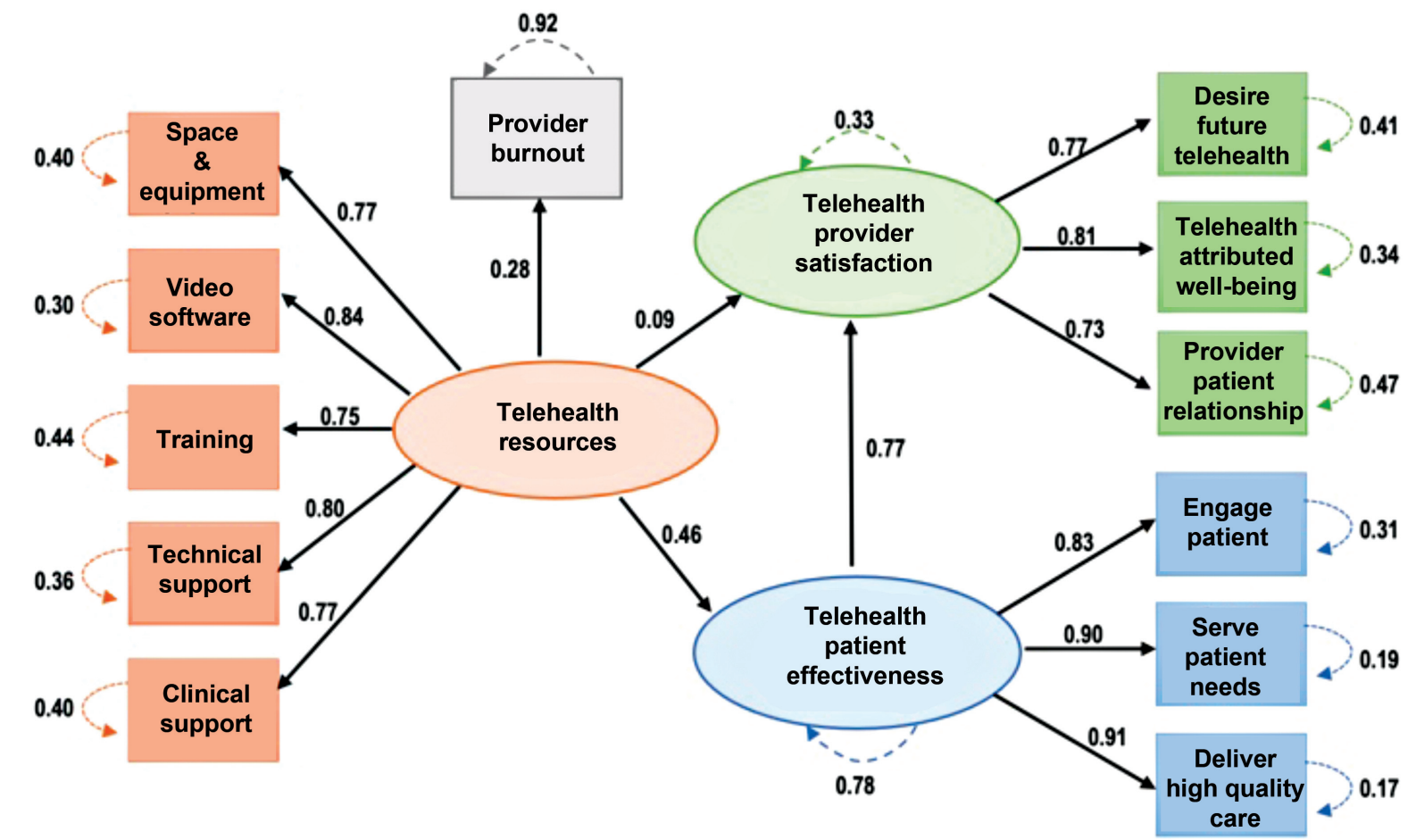

Fig. 3 Relationships between provider satisfaction, provider burnout, telehealth patient effectiveness and resources.

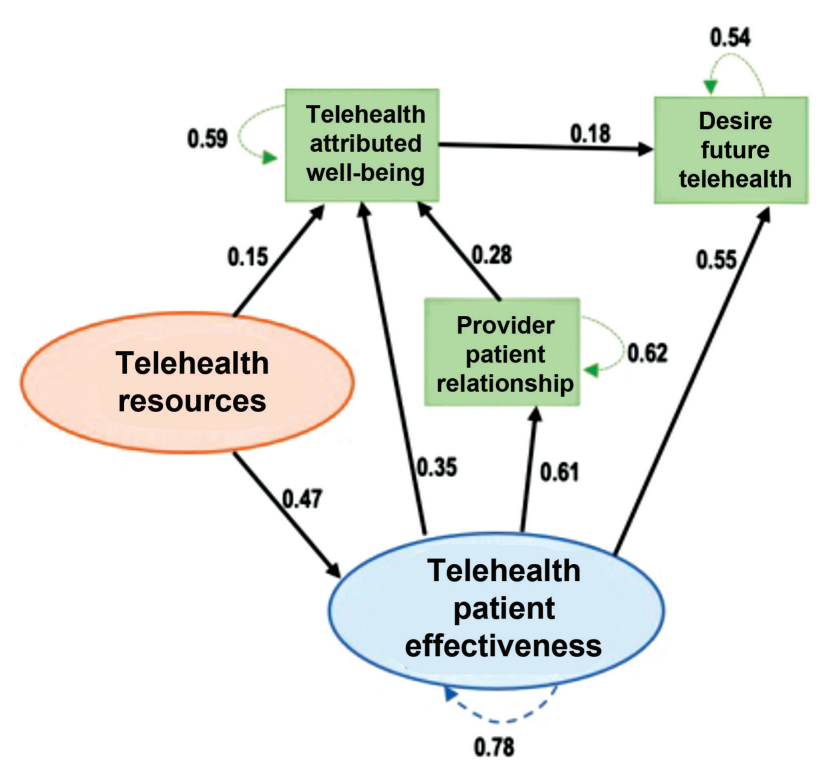

Fig. 4 Telehealth provider satisfaction model.

provider relationship and provider professional satisfaction. Notably, the importance of telehealth resources to provider well-being seems to be largely indirect, mediated by the relationship between resources, and perceived telehealth patient effectiveness.

Our study results confirm worrisome widespread stress among pediatric providers, as well as prevalent indications of early burnout. Though pre-COVID-19 burnout estimates are not available for our sample population, the overall rate of burnout (33\%) is compatible with existing literature. ${ }^{31}$ The single-item burnout measure used in this study, incorporated based on its validation as a stand-alone measure, inclusion in several major studies, and minimal respondent burden, is useful for clinical assessment but tends to under-identify burnout. ${ }^{32}$

Providers reporting burnout in this study were not ascribing such symptoms to telehealth, whereas they were specifically estimating improvement in well-being attributable to the rapid shift to telehealth. In our analysis, provider burnout was not directly related to telehealth effectiveness, but was instead directly related to telehealth resources. We interpret this to reflect burnout arising from perceived demands that chronically outweigh perceived resources. ${ }^{33}$ The high-residual variance in burnout ( 0.92 ) in our structural model indicates that there are other significant burnoutimpacting factors that this survey did not capture. Our model also underscores that clinician well-being is not merely the absence of burnout, and each is sensitive to specific determinants.

Health care organizations were obliged to accelerate implementations of telehealth solutions during the public health crisis, without the planning and stakeholder involvement that typically characterize such operational endeavors. Our findings highlight opportunities with actionable potential to sustain use of telehealth and advance provider wellbeing.

First, health care systems are likely to benefit from investing in robust telehealth training for provider and patient users to promote skills acquisition and enhancement. The National Consortium of Telehealth Resource Centers and groups including the American Board of Pediatrics and the American Association of Medical Colleges are developing 
telehealth competency standards and curricula for health professionals and students. ${ }^{34}$ Further research is needed to evaluate telehealth content and instructional modes such as e-learning and simulation in meeting the needs of adult learners. Patient-directed education efforts are also needed to prepare patients and families for digital literacy navigation. As virtual service offerings evolve, telehealth usability testing with at-risk patient populations should be considered.

Second, our data suggest both proactive and on-demand technical supports are important to successful telehealth programs. Connectivity and audio/visual performance issues are prevalent, pernicious challenges to telehealth success. Designing clinical processes to anticipate and troubleshoot technical issues and making staff available to resolve problems has potential to minimize disruption to care. Healthcare organizations must assess and respond to broadband access and other prerequisite challenges in the communities they serve to support initial and ongoing telehealth use.

Third, providers indicated clinical workflows require reengineering for telehealth-only, as well as hybrid telehealth/in-person, care models. While telehealth is at a disadvantage in detecting many physical examination findings and performing procedural interventions, management of diverse acute and chronic conditions can leverage a combination of in-person and virtual services, and providers are interested in accommodating both. In narrative comments, providers reported challenges with follow-up of tasks emerging from video visits, likely reflecting a need to optimize immature teamwork processes specific to telehealth. Providers also described challenges maintaining clinic momentum when telehealth and in-person visits are intermixed, noting different pacing of appointment types complicates transitions between patients. Attention should be given to decision tool development to guide modality choice, implementation of appropriate provider schedules and support staffing ratios to permit smooth throughput, and clear lines of health team communication and coordination during and after video visits. Providers reported prolonged performance of telehealth led to eye strain and screen fatigue. Virtual visit "bookending," scheduling telehealth appointments at the start or end of the day, might enable short blocks of consecutive video visits to achieve efficiency and productivity, as well as work-life flexibility.

\section{Limitations}

Our study findings should be interpreted considering key limitations. Statistical modeling was employed to explore plausible pathway associations, but experimental studies are required to establish causality among constructs. Certain survey variables were excluded from structural modeling because dichotomization produced low-frequency items that the analysis could not support. This study was conducted during the height of the COVID-19 pandemic. Telehealth was at times the only available modality for nonemergent care, possibly inflating ratings for service delivery, and patient engagement (compared with an implicit alternative of no/foregone care). Our sample did not permit meaningful subanalysis by extent of telehealth use. Selfreported outcome measures create potential for social desirability bias and recall error. Additionally, voluntary samples introduce self-selection bias. The majority of respondents practiced in multiple environments; however, we did not specifically assess whether telehealth was available or leveraged in all practice settings. Our results are most applicable to ambulatory pediatric providers and may not be generalizable to nonpediatric providers or other clinical settings. Finally, the pandemic disrupted providers' personal, as well as professional lives, although the former was not specifically investigated in our analysis.

\section{Conclusion}

System-based commitments to provider's well-being create resilient organizations. Evaluations of the recent telehealth surge and its lessons are beginning to accumulate and should inform data-driven and evidence-based best practices improvement efforts. This study offers evidence that telehealth can promote rather than detract from professional wellbeing for providers by enabling provision of high quality, patient-centric care and offering added flexibility and worklife balance to providers. We found widespread provider's desire to continue telehealth related to a positive perception of how effectively the medium met their patient's needs which, in turn, was influenced by how well an institution provided necessary technical and clinical resources for providers to do their jobs well. Optimizing telehealth structures and workflows to improve reliability, efficiency, and clinical excellence will benefit individuals and health care institutions.

\section{Clinical Relevance Statement}

Providers who transitioned to telehealth during the novel coronavirus disease 2019 (COVID-19) pandemic have a desire to continue telehealth. Associated factors for provider well-being in a telehealth care delivery model include provider-perceived telehealth effectiveness and adequacy of telehealth resources. Health systems can use these findings to optimize telehealth structures and workflow while planning for a postpandemic care delivery model.

\section{Multiple Choice Questions}

1. According to the structural equation model, what factor is associated with telehealth-attributable provider wellbeing?

a. Perceived effectiveness of telehealth

b. Working closely with a trainee

c. Working at an academic medical center

d. Being a subspecialist

Correct Answer: The correct answer is option a. The factors associated with telehealth-attributed provider 
well-being include provider-perceived effectiveness of telehealth and adequacy of telehealth resources.

2. The likelihood of improved telehealth-associated wellbeing was

a. Highest among providers who also reported one or more symptoms of burnout

b. Not statistically different between providers who endorsed and denied professional stress

c. Lower than worsened telehealth-associated well-being

d. Lower among providers of advanced career stage

Correct Answer: The correct answer is option b. Absent symptoms of burnout, there was no statistically significant difference in likelihood of improved telehealth-associated well-being between providers who denied and those who endorsed professional stress. Providers with any degree of burnout were significantly less likely to report improved telehealth-attributed well-being. Career stage was not associated with telehealth-associated wellbeing.

\section{Protection of Human and Animal Subjects}

The study was performed in compliance with the World Medical Association Declaration of Helsinki on Ethical Principles for Medical Research Involving Human Subjects and was reviewed by the Institutional Review Boards of all participating organizations: Connecticut Children's (registration no.: IRB00000703), Nationwide Children's Hospital (registration no.: IRB00000568), and East Tennessee Children's Hospital (registration no.: IRB00002221).

Funding

None.

Conflict of Interest

None declared.

\section{Acknowledgments}

We are grateful to all the participants and contributors who made this study possible, including the following individuals: East Tennessee Children's Hospital: Heather Radu, MD; Nationwide Children's Hospital: Jeffrey Hoffman, MD, Rajesh Ganta, Richard Hoyt, Sam Yang, MD, and Mounika Gunta; Connecticut Children's Hospital: Alex Lawson, Phillip Hopkins, Jeffrey Sargent, MBA, and Jung Park. None of these individuals was financially compensated for work outside their regular salaries.

\section{References}

1 Mann DM, Chen J, Chunara R, Testa PA, Nov O. COVID-19 transforms health care through telemedicine: evidence from the field. J Am Med Inform Assoc 2020;27(07):1132-1135

2 Wosik J, Fudim M, Cameron B, et al. Telehealth transformation: COVID-19 and the rise of virtual care. J Am Med Inform Assoc 2020;27(06):957-962

3 Eschenroeder HC, Manzione LC, Adler-Milstein J, et al. Associations of physician burnout with organizational electronic health record support and after-hours charting. J Am Med Inform Assoc 2021;28(05):960-966
4 Friedberg MW, Chen PG, Van Busum KR, et al. Factors affecting physician professional satisfaction and their implications for patient care, health systems, and health policy. Rand Health Q 2014;3(04):1

5 Kroth PJ, Morioka-Douglas N, Veres S, et al. Association of electronic health record design and use factors with clinician stress and burnout. JAMA Netw Open 2019;2(08):e199609

6 Gardner RL, Cooper E, Haskell J, et al. Physician stress and burnout: the impact of health information technology. J Am Med Inform Assoc 2019;26(02):106-114

7 Wu DTY, Xu C, Kim A, Bindhu S, Mah KE, Eckman MH. A scoping review of health information technology in clinician burnout. Appl Clin Inform 2021;12(03):597-620

8 Hartzband P, Groopman J. Physician burnout, interrupted. N Engl J Med 2020;382(26):2485-2487

9 Mehta S, Machado F, Kwizera A, et al. COVID-19: a heavy toll on health-care workers. Lancet Respir Med 2021;9(03):226-228

10 Reliford A, Adebanjo B. Use of telepsychiatry in pediatric emergency room to decrease length of stay for psychiatric patients, improve resident on-call burden, and reduce factors related to physician burnout. Telemed J E Health 2019;25(09):828-832

11 Chu-Weininger MY, Wueste L, Lucke JF, Weavind L, Mazabob J, Thomas EJ. The impact of a tele-ICU on provider attitudes about teamwork and safety climate. Qual Saf Health Care 2010;19(06):e39

12 Kuo KM, Talley PC, Lee CM, Yen YC. The influence of telemedicine experience on physicians' perceptions regarding adoption. Telemed J E Health 2015;21(05):388-394

13 Sauers-Ford HS, Hamline MY, Gosdin MM, et al. Acceptability, usability, and effectiveness: a qualitative study evaluating a pediatric telemedicine program. Acad Emerg Med 2019;26(09):1022-1033

14 Brigham T, Barden C, Dopp AL, et al. A journey to construct an allencompassing conceptual model of factors affecting clinician well-being and resilience. Accessed January 11, 2022: https:// nam.edu/wp-content/uploads/2018/01/Journey-to-ConstructConceptual-Model.pdf

15 Doarn CR, Pruitt S, Jacobs J, et al. Federal efforts to define and advance telehealth-a work in progress. Telemed J E Health 2014; 20(05):409-418

16 Weaver MS, Lukowski J, Wichman B, Navaneethan H, Fisher AL, Neumann ML. Human connection and technology connectivity: a systematic review of available telehealth survey instruments. J Pain Symptom Manage 2021;61(05):1042-1051.e2

17 deMayo R, Heggland A, Grindle C, et al. Pediatric telemedicine work and well-being survey. Accessed May 31, 2021: https:// is.gd/peditelemed

18 Arch collaborative example survey. Available athttps://klasresearch.com/images/pages/usability-studies/base-emr-experience-survey.pdf

19 Sisk B, Alexander J, Bodnar C, et al. Pediatrician attitudes toward and experiences with telehealth use: results from a national survey. Acad Pediatr 2020;20(05):628-635

20 Yip MP, Chang AM, Chan J, MacKenzie AE. Development of the Telemedicine Satisfaction Questionnaire to evaluate patient satisfaction with telemedicine: a preliminary study. J Telemed Telecare 2003;9(01):46-50

21 Parmanto B, Lewis AN Jr., Graham KM, Bertolet MH. Development of the Telehealth Usability Questionnaire (TUQ). Int J Telerehabil 2016;8(01):3-10

22 Bakken S, Grullon-Figueroa L, Izquierdo R, et al; IDEATel Consortium. Development, validation, and use of English and Spanish versions of the telemedicine satisfaction and usefulness questionnaire. J Am Med Inform Assoc 2006;13(06):660-667

23 Dolan ED, Mohr D, Lempa M, et al. Using a single item to measure burnout in primary care staff: a psychometric evaluation. J Gen Intern Med 2015;30(05):582-587

24 random.polychor.pa: A Parallel Analysis with Polychoric Correlation Matrices. Accessed May 31, 2021: https://CRAN. Rproject.org/package $=$ polycor 
25 Revelle W. psych: Procedures for Psychological, Psychometric, and Personality Research. R package version 2.1.3. Northwestern University: 2021 Accessed May 31, 2021 at: https://CRAN.Rproject.org/package=psych

26 Rosseel Y. lavaan: An R Package for Structural Equation Modeling.J Stat Softw 2012;48(02):1-36

27 West CP, Dyrbye LN, Sloan JA, Shanafelt TD. Single item measures of emotional exhaustion and depersonalization are useful for assessing burnout in medical professionals. J Gen Intern Med 2009;24(12):1318-1321

28 Brady KJS, Ni P, Carlasare L, et al. Establishing crosswalks between common measures of burnout in US physicians. J Gen Intern Med 2021. Doi: 10.1007/s11606-021-06661-4

29 Hair J Jr., Sarstedt M, Hopkins LG, Kuppelwieser V. Partial least squares structural equation modeling (PLS-SEM). Eur Bus Rev 2014;26(02):106-121
30 Fornell C, Larcker DF. Evaluating structural equation models with unobservable variables and measurement error. J Mark Res 1981; 18(01):39-50

31 Rotenstein LS, Torre M, Ramos MA, et al. Prevalence of burnout among physicians: a systematic review. JAMA 2018;320(11): $1131-1150$

32 West CP, Dyrbye LN, Satele DV, Sloan JA, Shanafelt TD. Concurrent validity of single-item measures of emotional exhaustion and depersonalization in burnout assessment. J Gen Intern Med 2012; 27(11):1445-1452

33 Demerouti E, Bakker AB, Nachreiner F, Schaufeli WB. The job demands-resources model of burnout. J Appl Psychol 2001;86 (03):499-512

34 AAMC. Telehealth competencies across the learning continuum. Accessed January 11, 2022 at: https://store.aamc.org/downloadable/download/sample/sample_id/412/ 